\title{
Resistively-Heated Microlith-based Adsorber for Carbon Dioxide and Trace Contaminant Removal.
}

\author{
S. Roychoudhury, D. Walsh \\ Precision Combustion, Inc.
}

\author{
J. Perry \\ National Aeronautics and Space Administration.
}

Copyright @ 2005 SAE International.

\begin{abstract}
An integrated sorber-based Trace Contaminant Control System (TCCS) and Carbon Dioxide Removal Assembly (CDRA) prototype was designed, fabricated and tested. It corresponds to a 1-person load. Performance over several adsorption/regeneration cycles was examined. Vacuum regenerations at effective timel temperature conditions, and estimated power requirements were experimentally verified for the combined $\mathrm{CO}_{2} /$ trace contaminant removal prototype. The current paper details the design and performance of this prototype during initial testing at $\mathrm{CO}_{2}$ and trace contaminant concentrations in the existing CDRA, downstream of the drier. Additional long-term performance characterization is planned at NASA. Potential system design options permitting associated weight, volume savings and logistic benefits, especially as relevant for long-duration space flight, are reviewed.
\end{abstract}

The technology consisted of a sorption bed with sorbentcoated metal meshes, trademarked and patented as Microlith by Precision Combustion, Inc. (PCl). By contrast the current $\mathrm{CO}_{2}$ removal system on the International Space Station employs pellet beds. Preliminary bench scale performance data (without direct resistive heating) for simultaneous $\mathrm{CO}_{2}$ and trace contaminant removal was reviewed in SAE 2004-012442. In the prototype, the meshes were directly electrically heated for rapid response and accurate temperature control. This allowed regeneration via resistive heating with the potential for shorter regeneration times, reduced power requirement, and net energy savings vs. conventional systems. A novel flow arrangement, for removing both $\mathrm{CO}_{2}$ and trace contaminants within the same bed, was demonstrated. Thus, the need for a separate trace contaminant unit was eliminated resulting in an opportunity for significant weight savings. Unlike the current disposable charcoal bed, zeolites for trace contaminant removal are amenable to periodic regeneration.

\section{INTRODUCTION}

Adsorption processes have been used for cabin air quality control on all crewed spacecraft, and is expected to continue to remain at the forefront of spacecraft cabin air quality control technologies. As mission durations increase and exploration goals reach beyond Earth orbit, the need for regenerable adsorption processes becomes paramount.

The TCCS removes trace chemical contaminants (e.g. alcohols, ketones, aromatics, $\mathrm{CO}$, halocarbons, hydrocarbons, and ammonia) from cabin air. The current TCCS on the International Space Station (ISS) uses a 50 Ib. bed of specially treated activated carbon and thermal catalytic oxidation. The fixed activated carbon bed is replaced periodically (>2 yrs).

The CDRA, located in the ISS, employs four fixed beds containing materials that first dry the process air and then remove the $\mathrm{CO}_{2}$ in separate unit operations. The drying mitigates the effect of water coadsorption on the $\mathrm{CO}_{2}$ adsorption process. The four beds alternate between adsorption and desorption cycles. Details on the CDRA process may be found in Reference 5 .

NASA-supported research at $\mathrm{PCl}$ has demonstrated that zeolites deposited on Microlith metal mesh elements can effectively adsorb a number of the contaminants of interest. The inert Microlith substrate and the use of a binder during deposition of the zeolites on them results in volumetric sorbent loadings lower than the carbon bed. However, the ability to directly resistively heat the metal mesh support allows rapid periodic regenerations [1]. This implies significantly reduced weight and volume for the current TCCS subassembly.

The potential of an integrated trace contaminant (TC) and $\mathrm{CO}_{2}$ removal within the same bed was identified and examined. Both TCCS and CDRA performance was demonstrated within the volume envelope of the current CDRA unit, eliminating the need for the current TCCS unit entirely. This permits weight, volume, and logistics 
savings and possibly offers power savings as well versus the current CDRA. Multi-cycle durability testing demonstrated a robust system. The data was used to design an electrically heated $\mathrm{CO}_{2} / \mathrm{TCCS}$ sorber prototype that was subsequently fabricated, tested, and delivered to NASA, for continued investigation.

\section{MICROLITH CATALYST TECHNOLOGY:}

The Microlith technology consists of a series of ultrashort-channel-length, low thermal mass coated metal meshes. It replaces the long channels of conventional monoliths with a series of short channel length substrates. Whereas in a conventional honeycomb monolith a fully developed boundary layer is present over a considerable length of the device, the very short channel length characteristic of the Microlith substrate avoids boundary layer buildup. Since heat and mass transfer coefficients depend on the boundary layer thickness, avoiding boundary layer buildup enhances transport properties. The Microlith can also pack more active surface area into a small volume, for increased adsorption area for a given pressure drop. The technology has been rigorously demonstrated in applications such as exhaust aftertreatment [2], space station air cleaning [3, 4], fuel processing [5] and catalytic combustion [6].

Zeolite adsorber preparation: Several formulations were evaluated in an effort to produce an adherent coating. The Microlith substrate geometry poses unique challenges in the development of adherent coatings. The coatings must be easily applied, have a high degree of adhesion and cohesion, and be sufficiently abrasion resistant to withstand routine handling. At the same time, the formulation must retain the desired chemical and physical characteristics of the zeolite. In general, zeolite powders with binder materials were applied to the Microlith substrate. The coatings were applied to the substrate using methods developed at $\mathrm{PCl}$ that allow for rapid application of ceramic coatings to large volumes of substrate. Electron microscopy of the zeolite coated Microlith, Fig. 1, 2, indicated uniform coatings with complete coverage. Cross-sectional views indicated that the coating was adherent and that the thickness of the coating was relatively uniform on all surfaces of the substrate.

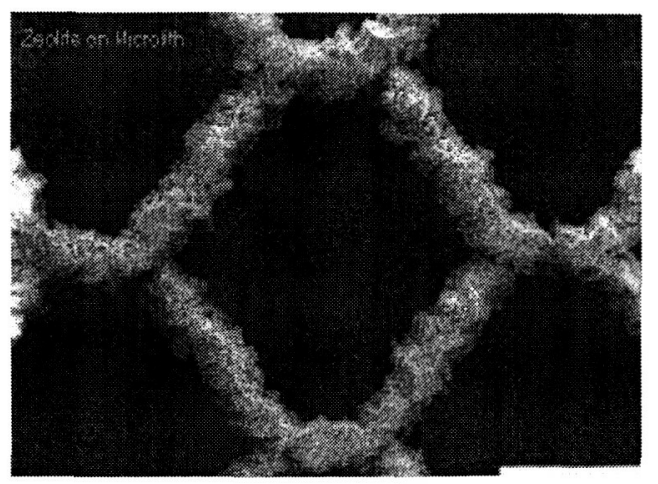

Figure 1: Surface scan SEM of zeolite coated Microlith.

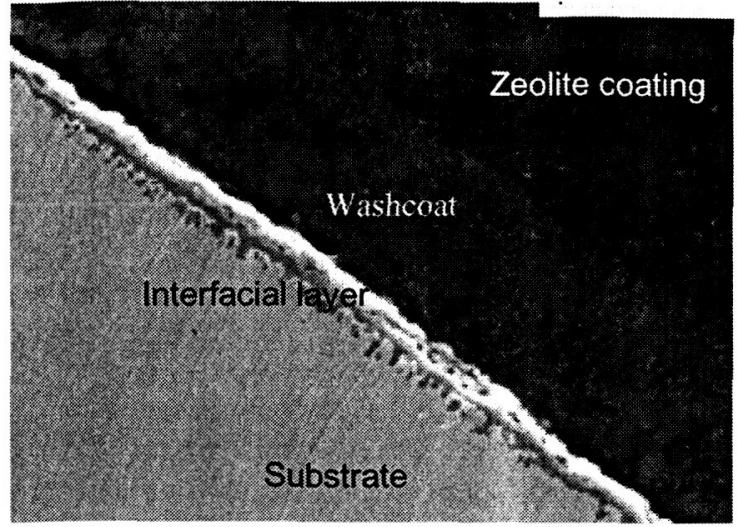

Figure 2: Cross sectional SEM view of the coated Microlith substrate.

\section{EXPERIMENTAL DETAILS:}

Durability Testing: Multi-cycle testing with both $\mathrm{CO}_{2}$ and all 5 trace components was done to examine robustness of the Microlith supported sorbents. Durability testing was conducted in a bench scale test rig. The testing employed a linear stack of screens, with external heating (rather that direct heating) during vacuum regeneration. The sorber housing had a thermocouple located to read the mid-bed centerline temperature. Since only a single thermocouple was reading was taken, axial and radial temperature uniformity could not be monitored. To reduce heat losses during regeneration, which could have accompanied the earlier use of a rope heater, the sorber housing, after isolation from the rest of the sorption unit, was subjected to vacuum and externally heated within a programmable oven. Bed regeneration temperatures (typically $\sim 230{ }^{\circ} \mathrm{C}$ ) were maintained for $\sim 90$ minutes after the thermocouple in the center of the bed registered the target value after which the oven cooled to room temperature over several hours. Vacuum was maintained on the sorbent housing during oven cool down. Prior to the first sorption cycle, the freshly charged unit was subjected to the modified vacuum regeneration procedure; regeneration was also conducted after each subsequent sorption cycle.

The reactor design employed alternate layers of coated Microlith and a similarly washcoated insulating material. Approximately $80 \%$ of the available volume was devoted to supported $5 \mathrm{~A}$ sorbent for $\mathrm{CO}_{2}$ removal, and $\sim 20 \%$ was used for supported $\mathrm{Y}$ and ZSM-5 for trace contaminant removal. The reactor was tested for 30 sorption/ regeneration cycles over 520 hours to demonstrate durability. The end of a sorption cycle was defined as the point at which any of the delivered feed components closely approached ( $-98 \%)$ its feed concentration. The feed concentrations were $\sim 1 / 2$ SMAC (Spacecraft Maximum Allowable Concentration) for all components with the exception of $\mathrm{NH}_{3}$ which was delivered at $75 \%$ $100 \%$ SMAC to facilitate analysis. Results of the 30 cycle durability testing are summarized in Figure 3 and Table 1 below. 


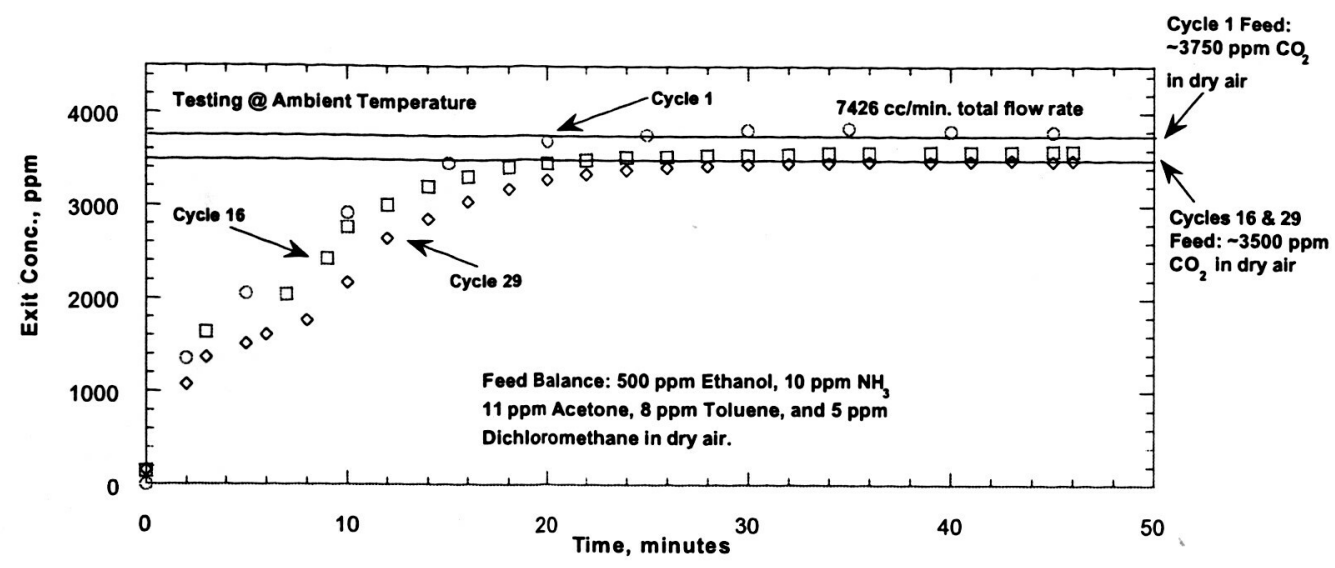

Figure 3: Sample durability data from cycles 1, 16 and 29.

Table 1: Sample durability data.

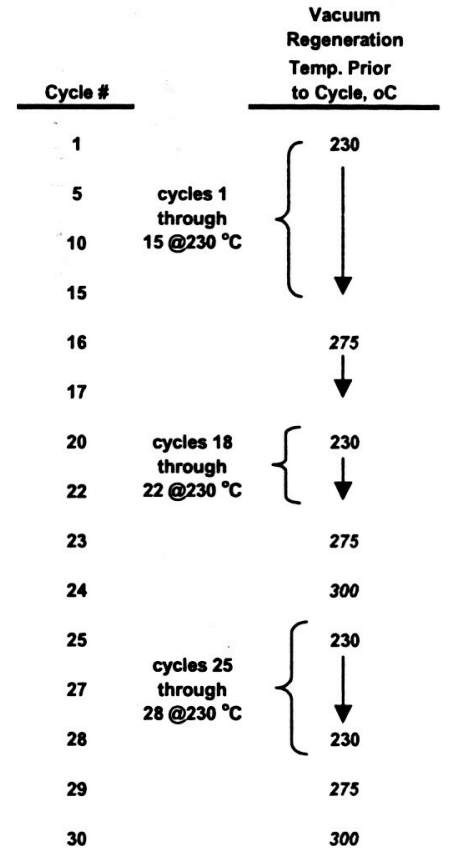

\begin{tabular}{c}
$\begin{array}{c}\text { Relative CO2 Sorp. } \\
\text { Performance }\end{array}$ \\
\hline 1.00 \\
0.72 \\
0.57 \\
0.58 \\
1.02 \\
1.37 \\
0.83 \\
0.35 \\
0.70 \\
1.25 \\
1.15 \\
0.50 \\
- \\
1.22 \\
\hline .50
\end{tabular}

\begin{tabular}{ccccc}
\multicolumn{5}{c}{ Ppm Trace Component @ CO2 End of Cycle } \\
\hline \hline Ethanol & Acetone & DCM & Toluene & $\mathrm{NH}_{3}$ \\
\hline 3 & 0 & 0 & 0 & 0 \\
4 & 0 & 0 & 0 & 0 \\
5 & 0 & 0 & 0 & 0 \\
24 & 0.2 & 0 & 0 & 0 \\
47 & 0.9 & 0 & $<0.1$ & 0 \\
52 & 1 & 0 & $<0.1$ & 0 \\
74 & 1.2 & 0.9 & 0.1 & 0 \\
65 & 0.8 & 0.9 & 0.1 & 0 \\
86 & 1.6 & 1 & 0.2 & 0 \\
48 & 0.8 & 0.2 & $<0.1$ & 0 \\
63 & 1 & 1.1 & 0.1 & 0 \\
80 & 1.3 & 1.1 & 0.1 & 0 \\
- & - & - & - & - \\
82 & 1.4 & 1.1 & 0.1 & 0 \\
80 & 1.4 & 1.3 & 0.2 & 0 \\
\hline & & & &
\end{tabular}

As the results shown above demonstrate (Table 6), $\mathrm{CO}_{2}$ breakthrough defined the end of cycle, i.e. trace contaminants were below their inlet concentration at the point where $\mathrm{CO}_{2}$ exit concentration approached the inlet concentration. Table 6 illustrates that vacuum regeneration at $230^{\circ} \mathrm{C}$ over the first 15 cycles resulted in decreasing $\mathrm{CO}_{2}$ sorption. This decline parallels that for trace contaminant testing using zeolite "sandwiches" using a $230{ }^{\circ} \mathrm{C}$ regeneration temperature. Since only a single point mid-bed temperature measurement was available, the possibility exists that internal gradients may have resulted in incomplete regeneration of the bed.

Regeneration at mid-bed temperatures above $230{ }^{\circ} \mathrm{C}$, however, was able to restore the lost $\mathrm{CO}_{2}$ sorption capacity, as illustrated by the performance in cycle 16 after a $275{ }^{\circ} \mathrm{C}$ regeneration. Back to back cycles regenerated at $275^{\circ} \mathrm{C}$ actually exceeded the sorption observed in cycle 1 (1.3x greater $\mathrm{CO}_{2}$ sorption). This is speculated to result from the creation of a cleaner, drier sorbent surface vs. that of the fresh sorbent which was

subjected to a single milder pretreatment at the $230{ }^{\circ} \mathrm{C}$ temperature. (An initial hot, dry $\mathrm{N}_{2}$ purge was not employed in these runs. Later experience indicated that such a treatment could expedite water removal from the adsorbent which was sorbed during reactor assembly. Higher temperature vacuum regeneration is likely gradually achieving this removal.)

Returning to the $230{ }^{\circ} \mathrm{C}$ regeneration temperature over the next 5 cycles (cycles 18 through 22), again results in declining cycle-to-cycle performance which appeared to be more extensive than that in cycles 1 through 5 . Again, back-to-back regenerations at higher temperatures in cycles 23 and $24\left(275^{\circ} \mathrm{C}\right.$ and $300^{\circ} \mathrm{C}$, respectively) resulted in $\mathrm{CO}_{2}$ sorption $1.25 \mathrm{x}$ greater than cycle 1 . Finally, performance declines over the next 4 cycles regenerating at $230{ }^{\circ} \mathrm{C}$ were once again overcome by back-to-back regenerations at the higher temperatures in cycles 29 and $30(1.2 x$ and $1.5 x$ greater $\mathrm{CO}_{2}$ sorption, respectively). 
These results indicated that the system was robust and suggested that an acceptable operating regimen could employ lower temperature regeneration (i.e. lower power requirement) over several sorption cycles in conjunction with periodic higher temperature regenerations to fully restore bed sorption capacity. This strategy is employed in the current pellet bed CDRA.

Over the 30 cycles, the trace contaminants remained at $<25 \%$ of their inlet concentrations at end-of-cycle. The exit concentrations increased as the number of cycles increased and showed some variation with regeneration temperature. Overall, however, the data suggested the attainment of an "equilibration" value with increasing number of cycles which - over the regeneration temperature range employed - was relatively invariant (Runs 20, 23, 27, 29, and 30).

\section{PROTOTYPE DESIGN AND FABRICATION.}

Implementing a design with a linear stack of screens presented difficulties with respect to ensuring electrical continuity over a large number of elements, avoiding shorting, achieving the desired resistance, edge sealing, assembly difficulties, etc. Furthermore, adequately addressing such issues would result in increased weight.

The use of a "jelly roll" coil in a radial flow configuration was chosen and implemented. The radial flow arrangement provided volumetric sorbent loadings at least comparable to a linear bank of screen elements. Furthermore, from the electrical and hardware assembly vantage points, a continuous length of coated screen largely mitigated the complicating issues encountered with a bank of screens. For these reasons, the prototype design was developed based on a two layer "sandwich" system consisting of a sorbent coated insulating layer and a sorbent coated Microlith screen layer (total coated thickness of the combined layers is $\sim 0.1 \mathrm{~cm}$ ). For dual function $\mathrm{CO}_{2}$ removal/TCCS, the appropriate lengths of the insulating and Microlith layers were coated with the desired amounts of the preferred sorbents as determined from bench scale examinations. Upon coiling the dual layer system around a centerline feed supply tube, the radial flow configuration resulted as depicted in Figure 4. Direct electrical heating of the metal Microlith substrate to regenerate the sorbent could be implemented more readily in this arrangement. Detailed views, including some nominal dimensions, are shown (Figures 5, 6).

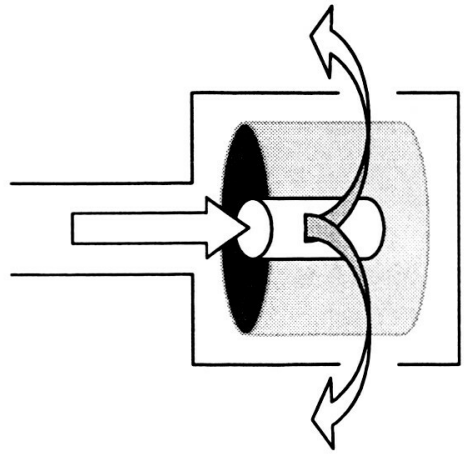

Figure 4: Radial Flow Sorber Configuration

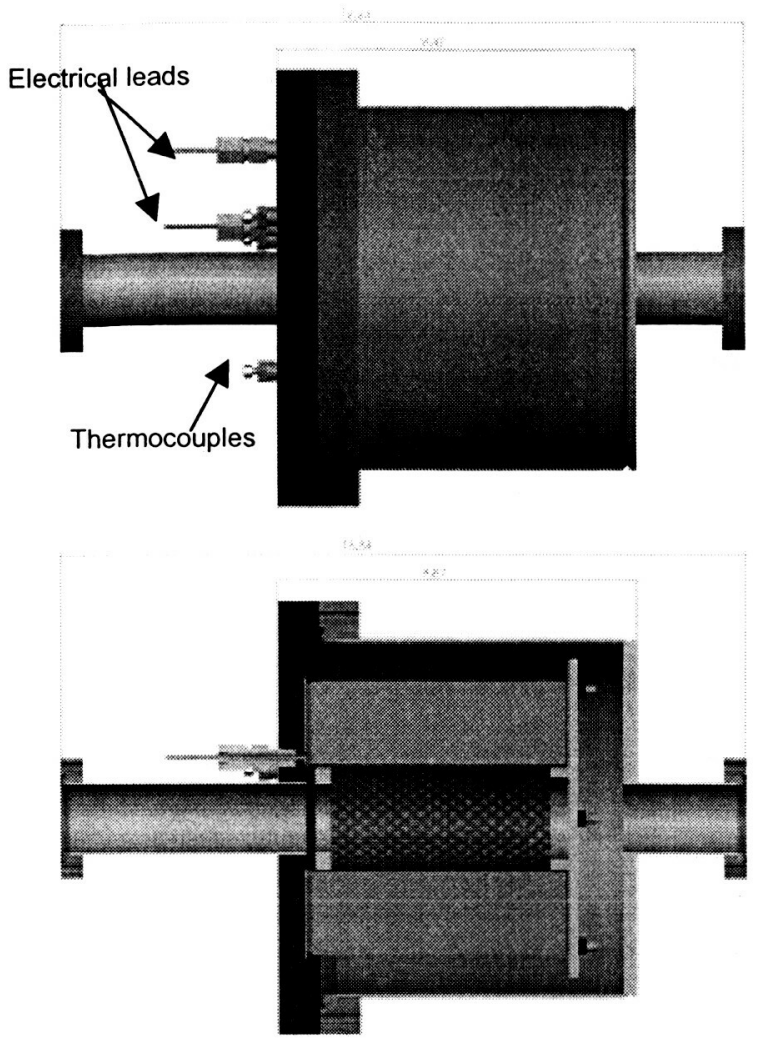

Figure 5, 6: Microlith Radial Flow Sorber - External and Internal Views.

The internal dimensions of the actual fabricated prototype sorber coil were $\sim 7.4$ " in diameter and $\sim 7.2$ " long, the centerline of which was occupied by a 3" diameter feed tube. The unit was targeted to operate at approximately the same nominal contact time as the current CDRA sorber $(\sim 1.8 \mathrm{sec}$.) This corresponded to a volumetric flow rate of $\sim 5 \mathrm{cfm}$ through the annular sorber coil volume of 258.5 in $^{3}$ (4235 cc). This flow rate corresponds to $1 / 4$ scale vs. the current CDRA which operates at a $20 \mathrm{cfm}$ throughput. The sorber coil plus the 3 " centerline feed tube occupied $\sim 5075 \mathrm{cc}$, or $\sim 30 \%$ of the sorbent volume in one of the canisters employed in the current CDRA. The annular coated coil itself occupied $-26 \%$ of the current CDRA sorbent volume. Deducting the feed tube volume of $\sim 840 \mathrm{cc}$ resulted in a net volume for the total sorber coil $\left(\mathrm{CO}_{2}\right.$ \& trace contaminant removal) of $4235 \mathrm{cc}$. Devoting $82.5 \%$ of this net sorber volume to $\mathrm{CO}_{2}$ sorption corresponded to 213.2 in $^{3}$ (3494 cc) of sorber volume.

The $\mathrm{CO}_{2}$ sorber regeneration requirement sets the cycle time for the TCCS sorption section of the coil, which occupied $17.5 \%$ of the total annular sorption volume or $45.1 \mathrm{in}^{3}$ (740 cc). The TCCS coating followed the $5 \mathrm{~A}$ coating and therefore was located at the end of the screen, i.e. at the outer portion of the wound coil. The initial $57.5 \%$ of this TCCS segment was washcoated with zeolite $Y$ and the balance with ZSM-5. Assuming no contribution to trace contaminant removal from the $5 \mathrm{~A}$ molecular sieve, and based on the data from the prior studies, this allocation of $15-20 \%$ of the sorber volume to a post-5A TCCS function (Y/ZSM-5) was estimated to be 
sufficient to maintain the exit concentration of the trace contaminants below their inlet levels during the sorption cycle.

Power requirements were examined for direct electrical heating of the coil based on estimates of the total energy required to heat the mass of sorber components and to supply the heat of desorption (dominantly that of $\mathrm{CO}_{2}$, but also that of the trace components). Radiation losses were ignored since these are likely to be small at the target regeneration temperatures for a well insulated system having a low ratio of external surface area to total sorber volume. Assuming no other losses, the total required energy during heat up to the regeneration temperature is the sum of $\mathrm{mC}_{\mathrm{p}} \Delta \mathrm{T}$ for the washcoat, substrate, and insulating layer plus the heats of desorption. The Microlith sorber coil has a lower bulk density vs. a pellet bed of $5 \mathrm{~A}(\sim 65 \%$ of the weight of an equivalent pellet volume), and a lower overall estimated specific heat. Assuming a $45 \mathrm{~min}$. sorption/regeneration cycle - 110 - 150 watts would be required per module depending upon the final target regeneration temperature $\left(230-300^{\circ} \mathrm{C}\right)$.

In the interest of time and cost, readily available off-theshelf - but relatively massive -vacuum rated flanges were used. Figure 7 shows the prototype during assembly, the dual layer wrapped coil, and the $3^{\text {" }}$ perforated feed tube around which the coil was wound. The weight of the adsorbent coil, thermocouples, bus bars and feed tube was $2.3 \mathrm{~kg}$. The completed prototype is shown in Figure 8. The flanges and housing weight was $-30 \mathrm{~kg}$, significantly increasing the heat loss component. In reality, dedicated flanges/housing would drastically reduce the weight.

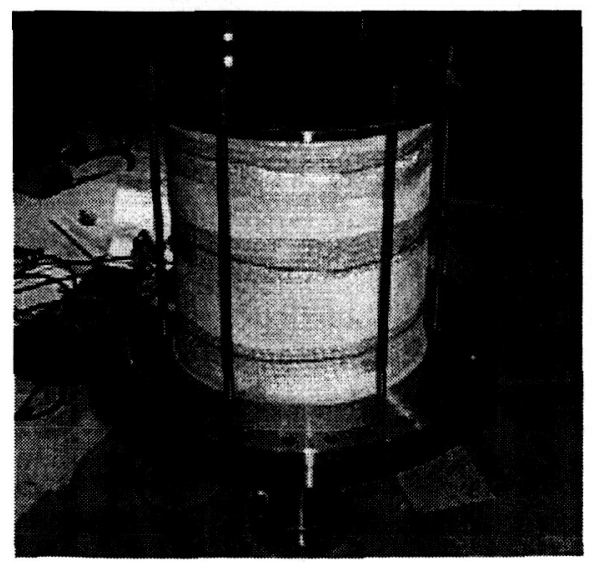

Figure 7: Prototype during assembly.

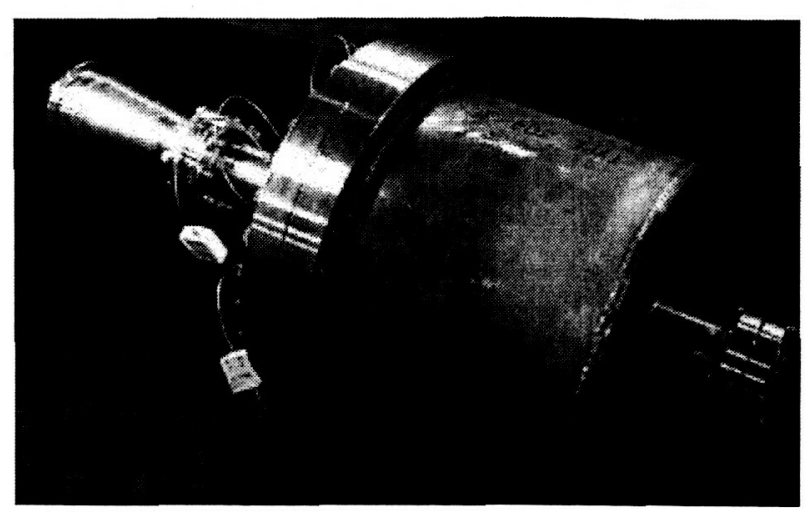

Figure 8: Completed prototype assembly.

\section{PROTOTYPE TESTING}

To gain experience with how the unit would respond when employing direct electrical heating of the Microlith substrate, pretreatment heating under vacuum of the fresh sorbent and vacuum regeneration of the saturated sorbent coil were performed more conservatively than calculations indicated was necessary. The unit was heated under vacuum until the lowest registering thermocouple reached $\sim 230-240{ }^{\circ} \mathrm{C}$. Temperature gradients within the unit were such that the highest registering thermocouple was usually $\sim 70-90{ }^{\circ} \mathrm{C}$ hotter $\left(\sim 300-320^{\circ} \mathrm{C}\right)$. After a 90 -minute hold, the unit was cooled under vacuum to room temperature prior to conducting a sorption cycle. Total flow rate was $\sim 5 \mathrm{cfm}$ and component concentrations were like those employed in the prior tasks $(-3500 \mathrm{ppm} \mathrm{CO}, 500 \mathrm{ppm}$ ethanol, 10 ppm $\mathrm{NH}_{3}, 11 \mathrm{ppm}$ acetone, $8 \mathrm{ppm}$ toluene, and $5 \mathrm{ppm}$ dichloromethane). Figure 9 presents the results from the first 3 prototype testing cycles. For vacuum regenration, the terminals were connected to a wall $A C$ power supply regulated by a variac. 


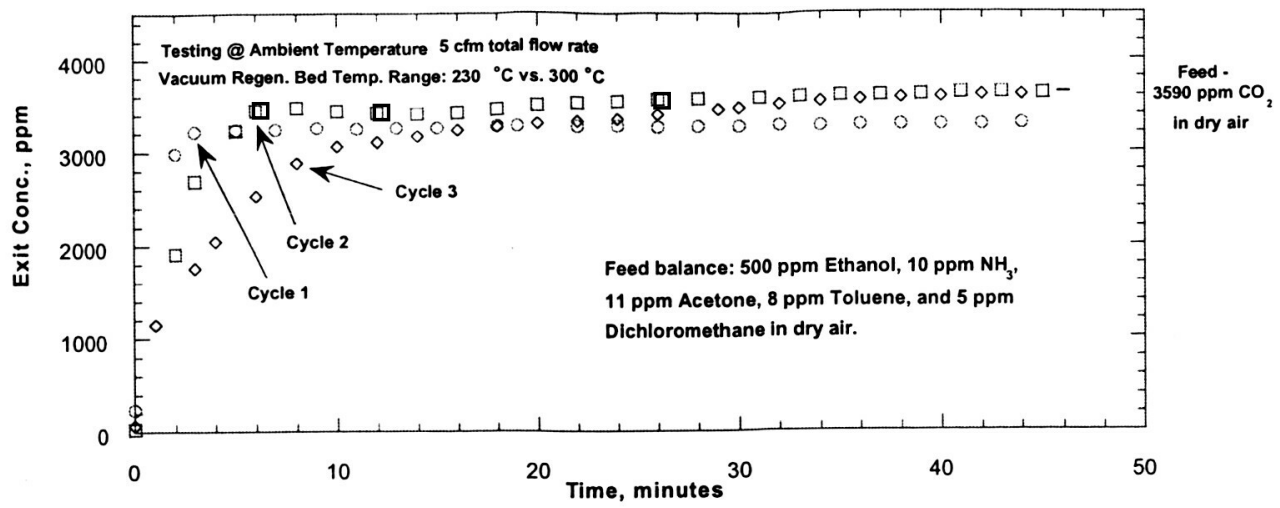

Figure 9: Prototype testing: cycle-to-cycle variations in $\mathrm{CO}_{2}$ sorption for cycles $1-3$.

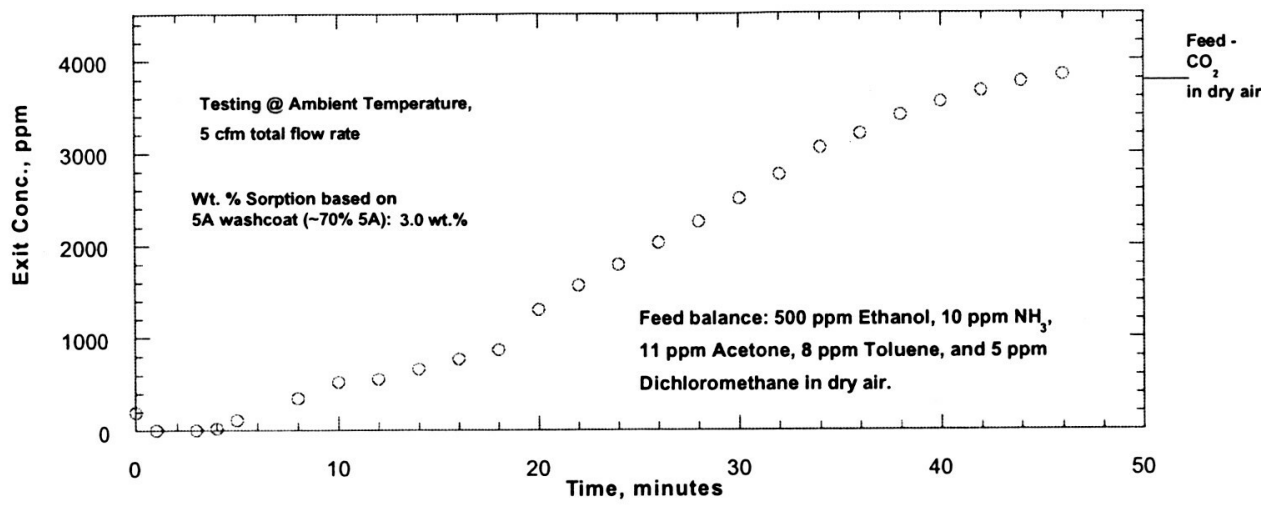

Figure 10: $\mathrm{CO}_{2}$ sorption for cycle 5 .

The data in the figure indicate poor performance but improving from cycle to cycle, suggesting a potentially lengthy process to remove water and/or other sorbed species to establish a dry, clean surface effective for sorption. After the fourth cycle, the prototype was regenerated more aggressively to accelerate the process so that performance could be demonstrated and assessed prior to the conclusion of the program. Consequently, after the 90 minute vacuum regeneration as described above, the bed was conditioned by removing moisture adsorbed during assembly by flowing dry nitrogen through the unit at $\sim 5 \mathrm{slpm}$ for $\sim 4.5$ hours at regeneration temperature. Thereafter, the unit was allowed to cool overnight in the dry nitrogen flow. Sorption cycle 5 was then performed, the results of which are shown in Figure 10.

The cycle 5 data indicate 3 wt $\% \mathrm{CO}_{2}$ sorption capacity and a $45-50$ minute cycle during which $\sim 55 \%$ of the delivered $\mathrm{CO}_{2}$ was sorbed. The marked improvement in $\mathrm{CO}_{2}$ sorption vs. the first 3 cycles underscored the need to establish the correct initial sorber suface conditions (dryness) to achieve proper performance. At $3 \mathrm{wt} \% \mathrm{CO}_{2}$ sorption, the prototype produced the best result observed during the study, suggesting that the performance observed in some of the earlier data may have been influenced by incompletely dried $5 \mathrm{~A}$ sorbent. As in the durability studies, trace contaminants in the exit gas at end of cycle were well below their inlet levels. With the exception of ethanol, trace contaminant levels were zero. The ethanol level was only $5 \mathrm{ppm}$ or $1 \%$ of its inlet concentration. While these end of cycle trace contaminant levels might rise over multi-cycle operation of the prototype, the durability studies suggest that they will equilibrate at levels appreciably lower than their inlet concentrations. Measurements indicated that the unit's pressure drop was $<0.5^{\prime \prime}$ of water.

Although the cycle 5 data indicated a 45-50 minute cycle time, one possible operating strategy could involve ending the cycle at $\sim 30$ minutes. At that point, less total $\mathrm{CO}_{2}$ will have been delivered, but $\sim 76 \%$ of the delivered amount will be sorbed, corresponding to $\sim 20 \mathrm{gms}$. Like the current CDRA, each Microlith based module would have a companion unit - the pair alternating between sorption and regeneration - and, therefore, each pair would sorb $\sim 40 \mathrm{gms} \mathrm{CO}_{2} / \mathrm{hr}$. At a metabolic generation rate of $\sim 2.2 \mathrm{lbs} /$ day of $\mathrm{CO}_{2} /$ person $(41.6 \mathrm{gm} / \mathrm{hr}$ ), one module pair would be required per person, and thus the total number of such module pairs employed would be determined by the crew size.

After cycle 5, the unit was subjected to the vacuum regeneration procedure noted above with direct heating of the Microlith substrate. The temperature vs. time history for this treatment is shown in Figure 11 along with the average power applied at various regeneration intervals. 


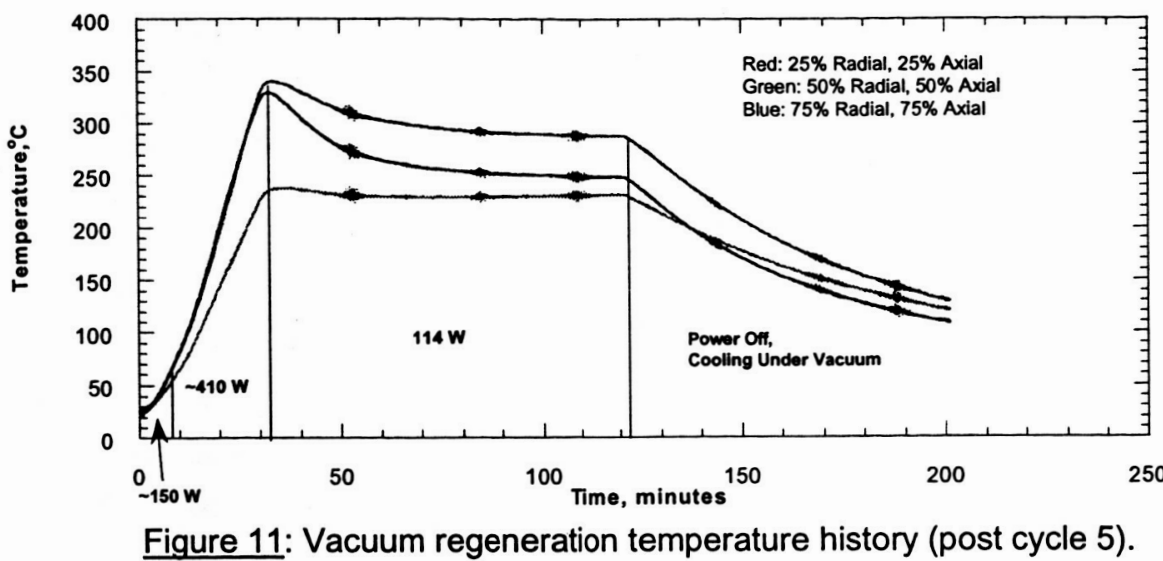

The data in Figure 11 indicates that heating to target regeneration temperatures can be achieved in $<35$ minutes. Potential heating times are likely much lower since a relatively conservative approach was still being exercised in this regeneration treatment. Power levels corresponding to the regions in the figure exceed the estimates discussed earlier which were based only on the substrate and sorbent masses, as well as desorption heat requirements, without consideration for radiative or conductive losses to the housing. Such losses were present in the prototype, the mass of which provided a larger heat sink than would be the case in an optimized future configuration. The total power requirement for a Microlith based system vs. the current CDRA will depend upon the number of module pairs employed, the characteristics of a modified housing within which a module is contained, and the heating rate and regeneration "soak time" (if any) employed. Present uncertainties associated with these variables preclude a definitive comparative assessment at this time. However, the calculations based on the characteristics of the two types of sorbent beds themselves suggest a reasonable likelihood that the final embodiment of a Microlith system will require $\leq 960 \mathrm{~W}$ employed in the current CDRA.

As noted above, depending upon the number of module pairs needed for a mission, the total volume of the Microlith based system might exceed that of the current CDRA. However, because of the lower bulk density of the Microlith supported sorbents, no sorbent weight penalty vs. the current CDRA should result for a crew of 6 or less. As with the power comparison, a total weight comparison vs. the current CDRA will depend upon the details of the housing weight, inlet and outlet lines, flange connections etc. in any final embodiment of the Microlith approach. The integrated CDRATTCCS Microlith based system, of course, would provide a baseline weight (and volume) savings by eliminating the current TCCS unit.

\section{CONCLUSIONS.}

The potential for a single Microlith based regenerable sorber unit to remove both $\mathrm{CO}_{2}$ and trace contaminants was demonstrated. Accompanying weight and volume savings arising from the possible elimination of the current TCCS charcoal bed ( $50 \mathrm{lbs})$ and a potentially lower weight for the Microlith unit versus the current CDRA was shown. The lower heat capacity $\left(C_{p}\right)$ for the Microlith unit is likely to result in power savings and indicated faster heat up. Since the Microlith unit can be regenerated quickly and more frequently than the current CDRA, weight, volume and power savings need to be quantified via a rigorous system analysis. Improved coatings were developed with further room for improvement. The durability of the coatings was shown to be stable over 500 hours of operation over 30 adsorption/regeneration cycles. The electrically, direct resistively, heated prototype showed stable and expected performance during qualification testing. A simple, flexible control system was used for regeneration (adaptable to available power). A modular unit with very low pressure-drop was demonstrated and delivered to NASA.

Potential Future Work: The results suggest a number of topics for possible additional study. These include, regeneration studies to define prototype performance as a function of heating rate, regeneration temperature, and "soak time" at temperature. For example, can rapid heating to $275{ }^{\circ} \mathrm{C}$ with no soak provide acceptable performance? Results could influence cycling strategy and its impact on power requirements. Potential tradeoffs between cycle time, unit size, and power consumption might lead to a smaller Microlith based system cycling more frequently. Multi-cycle durability studies using the prototype itself would be a desirable complement to the sub-scale durability tests. Further examination of axial flow coiled Microlith sorber configurations may lead to additional design options.

\section{ACKNOWLEDGMENTS}

We gratefully acknowledge the National Aeronautics and Space Administration's support in conducting a significant portion of this research. Any opinions, findings, and conclusions or recommendations expressed in this publication are those of the authors and do not necessarily reflect the views of NASA. The authors are also grateful to the technical support group at $\mathrm{PCl}$. Their skilful help made this work possible. 


\section{REFERENCES}

1. S. Roychoudhury, D. Walsh, J. Perry; Microlith Based Sorber for Removal of Environmental Contaminants; SAE 2004-01-2442

2. J. Bianchi, G. Muench, W.C. Pfefferle SAE 971023 , SAE Intl, 1997.

3. R. Carter, J. Bianchi, W. Pfefferle, S. Roychoudhury, J.L. Perry, SAE 972432, SAE Intl, 1997.

4. J.L. Perry, R.N. Carter and S. Roychoudhury, SAE 1999-01-2112, SAE International, (1999).
5. "A Compact Catalytic Combustor System for Small Turbogenerators." EC-Vol. 5, Proceedings of the International Joint Power Generation Conference.

6. "Performance of Microlith Based Catalytic Reactors for an Iso-octane Reforming System". SAE 2003-011366. M. Castaldi, S. Roychoudhury, et al.

\section{CONTACT}

* Consultant to $\mathrm{PCl}$.

Author for correspondence: Subir Roychoudhury, D.E.; Email: sroychoudhury@precision-combustion.com 